\title{
A Chloroplast phylogeny of Arisaema (Araceae) ILLUSTRATES TERTIARY FLORISTIC LINKS BETWEEN Asia, North America, and East Africa ${ }^{1}$
}

\author{
SusAnNe S. RenNer, ${ }^{2,4}$ Li-Bing Zhang, ${ }^{2,5}$ AND Jin MuratA ${ }^{3}$
}

\author{
${ }^{2}$ Department of Biology, University of Missouri-St. Louis, St. Louis, Missouri 63121 USA; The Missouri Botanical Garden, \\ St. Louis, Missouri 63166 USA; and \\ ${ }^{3}$ Botanical Gardens, The University of Tokyo, Tokyo 112-0001 Japan
}

\begin{abstract}
The evolution of Arisaema is reconstructed, based on combined sequences (2048 aligned bases) from the chloroplast trnL intron, trnL-trnF spacer, and rpl20-rps 12 spacer obtained for species from all 11 sections, including sectional type species and geographically disjunct East African and North American/Mexican species. Analyses were rooted with a representative sample of the closest outgroups, Pinellia and Typhonium, to rigorously test the monophyly of Arisaema. Sections in Arisaema are mostly based on leaf, stem, and inflorescence characters and, with one exception, are not rejected by the molecular data; however, statistical support for sectional relationships in the genus remains poor. Section Tortuosa, which includes eastern North American A. dracontium and Mexican A. macrospathum, is demonstrably polyphyletic. The third New World species, A. triphyllum, also occurs in eastern North America and groups with a different Asian clade than do A. dracontium/A. macrospathum. The genus thus appears to have entered North America twice. Fossil infructescences similar to those of A. triphyllum are known from approximately 18 million-year-old deposits in Washington State and can serve to calibrate a molecular clock. Constraining the age of A. triphyllum to 18 million years (my) and applying either a semiparametric or an ultrametric clock model to the combined data yields an age of approximately 31-49 my for the divergence of A. dracontium/A. macrospathum from their Asian relatives and of 19-32 my for the divergence between African A. schimperianum and a Tibetan/Nepalese relative. The genus thus provides an example of the Oligocene/Miocene floristic links between East Africa, Arabia, the Himalayan region, China, and North America. The phylogeny also suggests secondary loss of the environmental sex determination strategy that characterizes all arisaemas except for two subspecies of $A$. flavum, which have consistently bisexual spathes. These subspecies are tetraploid and capable of selfing, while a third subspecies of A. flavum is diploid and retains the sex-changing strategy. In the molecular trees, the sex-changing subspecies is sister to the two non-sex-changing ones, and the entire species is not basal in the genus.
\end{abstract}

Key words: Areae; Arisaema; Beringia; biogeography; molecular clock; Pinellia; sex change; Typhonium.

Arisaema comprises about 150 species of forest understory herbs (Murata, 1984; Gusman and Gusman, 2002; Li et al., in press). About 140 occur in the Himalayas, southern India, Sri Lanka, China, Korea, Japan, and Southeast Asia; one species ranges from Nepal, across Saudi Arabia and Oman to East Africa; five or six species are endemic in East Africa; and three are endemic to North America (Jack-in-the-pulpit, green dragon, and a close relative; Fig. 1). Arisaema typically occurs in cool temperate environments, including montane grasslands, with a few species thriving near timberline at $4200-4500 \mathrm{~m}$ in the Himalayas and at $3200 \mathrm{~m}$ in East Africa (on Mt. Ruwenzori). As expected from the geographic range of the genus, most species are deciduous and overwinter via underground tubers or rhizomes. Only about 38 species are evergreen. The sexually produced diaspores are berries that are spread by birds, and asexual propagation occurs via tuber offsets, sto-

\footnotetext{
${ }^{1}$ Manuscript received 11 September 2003; revision accepted 22 January 2004.

The authors thank T. Barkman, J. Bogner, P. Bruggeman, A. M. Chambers, Binghui Chen, G. Gusman, W. Hetterscheid, B. W. Magrys, J. McClements, H. He, C. Obunyali, P. Schmidt, J. W. Waddick, J. C. Wang, and the botanical gardens of Mainz and Munich for leaf material; B. Genton and A. Weerasooriya for help with sequencing; and G. Gusman for support throughout this study - without his material, this work would not have been possible. Funding for this research came from the University of Missouri system and the University of Missouri-St. Louis.

${ }^{4}$ Present address: Systematische Botanik, Ludwig-Maximilians-Universitaet, Menzinger-Str. 67, D-80638 Muenchen, Germany (renner@umsl.edu).

${ }^{5}$ Present address: Department of Biology, Colorado State University, Fort Collins, CO 80523.
}

lons, or rhizomes. Pollination usually is by fungus gnats (Mycetophilidae) and sciarid gnats (Sciaridae), but pollination by nectar-seeking small bees may also play a role (Murata et al., 1993; Vogel and Martens, 2000).

Arisaema is notorious for its interannual "sex change" and is the only Araceae, and one of very few angiosperms, that has labile sex determination. Environmental, or labile, sex determination, is a life history strategy in which sex is determined by the environment and may change during an individual's lifetime (Charnov and Bull, 1977; Freeman et al., 1980). It evolved an unknown number of times in animals and plants, with Arisaema one of the best-documented cases in plants (Schlessman, 1988). Sex expression in Arisaema is dependent on nutrient status, with several reversals possible during a plant's life. Large plants produce staminate and pistillate flowers or only pistillate flowers and function as hermaphrodites or females. Small plants produce only staminate flowers. As demonstrated experimentally, and well-known to Arisaema growers, size (thus sex) is influenced by resources accumulated in the root storage organs, with effects carrying over for several years and species apparently very long-lived (20 years or more; Bierzychudek, 1984).

All Arisaema are sex changers, although literature before 1990 sometimes cited A. flavum as consistently producing male and female flowers simultaneously (bibliography available at http://www.umsl.edu/ biosrenn/). However, Murata (1990c) discovered sex-changing populations of A. flavum, described as subspecies tibeticum, leaving the remaining two subspecies of A. flavum as the only known non-sex-changing 


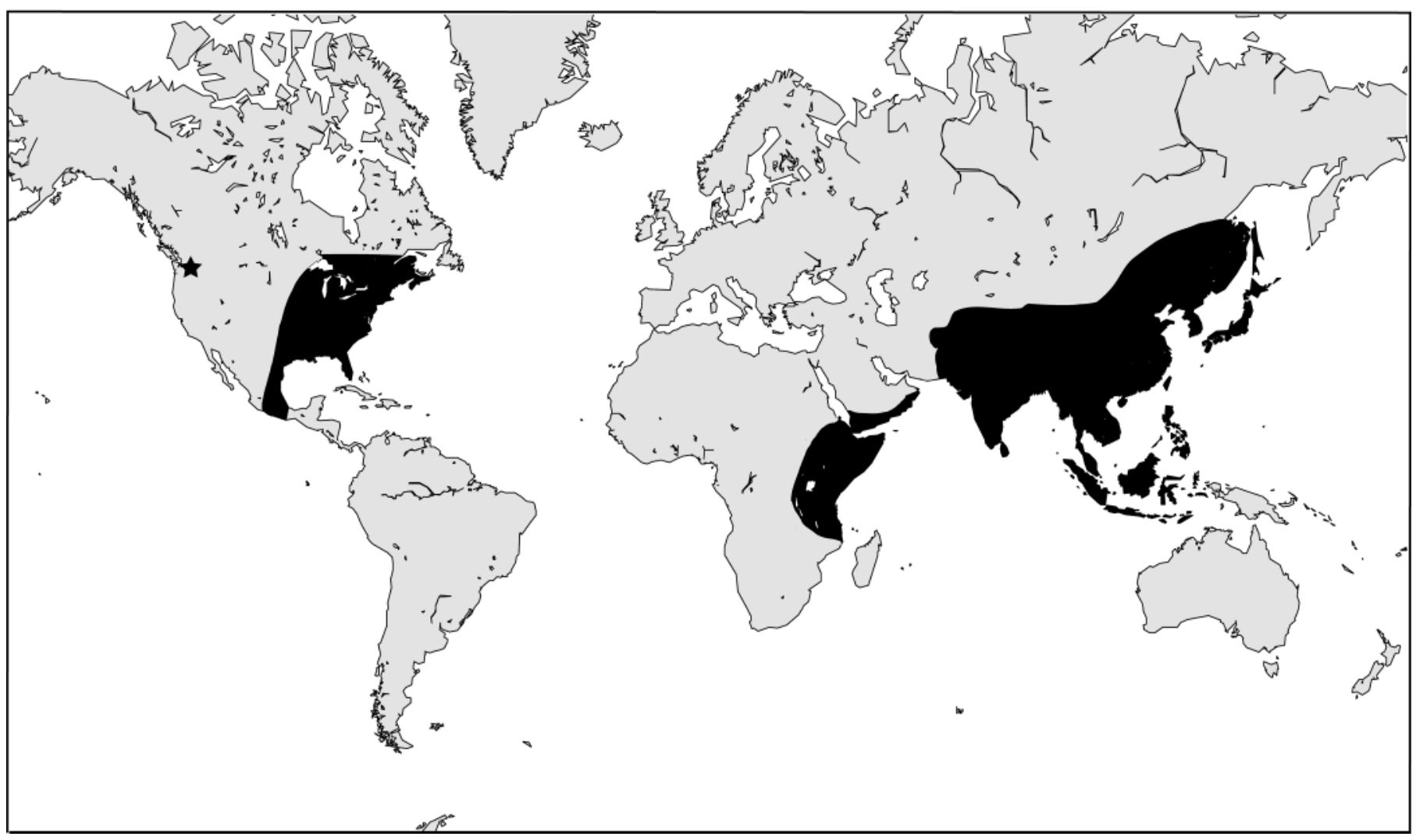

Fig. 1. Distribution map of Arisaema. The star indicates the locality (Spokane) where an A. triphyllum-like fossil infructescence has been found.

entities in Arisaema. Remarkably, subspecies tibeticum is diploid, while the two non-sex-changing subspecies are tetraploid and set viable seeds after selfing (automatic selfing is prevented in sex-changing species of Arisaema). This suggests a return from environmental sex determination to simultaneous bisexuality concomitant with polyploidy and regular selfing. Because of its unusual male flowers (with a single stamen vs. the usual 3-5) and the absence of sex change in two of its three subspecies, A. flavum has been seen as "phenetically most primitive" (Grayum, 1990), while others have seen it as derived (Li, 1981; Vogel and Martens, 2000). The monophyly and phylogenetic position of this species and the possibility of a secondary loss of environmental sex determination are among the questions investigated here.

Except for its unusual sexual strategy, Arisaema shares its reproductive and vegetative characters with Pinellia, a genus of six species in temperate East Asia (G. Zhu and H. Li, unpublished manuscript), and Typhonium, a heterogeneous assemblage of about 50 species from tropical Asia, southeast Australia, and tropical Africa (T. venosum; Sriboonma et al., 1994; Hetterscheid and Boyce, 2000; Hetterscheid et al., 2001; species numbers are moot since Typhonium is paraphyletic; Renner and Zhang, in press). At least 10 species have been transferred between these genera, for example, A. hirsutum S. Y. Hu [Typhonium hirsutum (S. Y. Hu) J. Murata et Mayo], A. submonoicum Gagnep. [T. horsfieldii (Miq.) Steenis], and A. tripartitum Engl. [Pinellia tripartita (Blume) Schott], illustrating the genera's indistinct boundaries. Testing the monophyly of Arisaema was therefore another important goal of our study.
Morphological and biogeographic work on Arisaema has suggested that it reached East Africa from Asia (Li, 1981; Grayum, 1990) and that it entered North America twice. The latter is implied by the traditional placement of the American species in different sections. (The status of the Mexican species, A. macrospathum Benth., as either a distinct species or as a subspecies of $A$. dracontium has long been unclear [but see Gusman, 2000].) In spite of their placement in different sections, the North American species are thought to hybridize (Sanders and Burk, 1992; K. Clay, Indiana University, personal communication), perhaps arguing for relatively recent divergence. The African and Arabian species of Arisaema have been revised (Mayo and Gilbert, 1986), but remain poorly collected, and their sectional assignment has been difficult (Murata, 1984, 1990b). Mayo and Gilbert (1986; also Mayo, 1993) have stressed the morphological similarities that exist between African/Arabian and Indian/Nepalese species pairs; they specifically mention the species pair A. consanguineum (Himalaya, northern Thailand) and A. mildbraedii (Africa). Although our sampling of African species is limited, we were able to obtain material of this particular pair and thus could test Mayo and Gilbert's hypothesis.

To better understand the relationships of Asian Arisaema to species occurring in Africa and North America and to gain insight into the evolutionary plasticity of the sex change strategy and the leaf and inflorescence characters that form the basis for current classifications (Hara, 1971; Murata, 1984, 1990b; Gusman and Gusman, 2002; Li et al., in press), we here analyze chloroplast sequences obtained for 81 accessions 
representing 77 of the approximately 150 recognized species of Arisaema.

\section{MATERIALS AND METHODS}

Taxon sampling, DNA isolation and amplification, and sequence alignment-The Appendix (see Supplemental Data accompanying the online version of this article) lists all species sequenced for this study, with sources and GenBank accession numbers. The samples include 81 accessions of Arisaema, five of Pinellia, and nine of Typhonium. A study based on mitochondrial and chloroplast DNA and including representatives of the 16 genera in the larger clade to which Arisaema belongs was unable to resolve the trichotomy of Arisaema, Pinellia, Typhonium (Renner and Zhang, in press). We therefore rooted our trees with a representative sample of species of Pinellia and $T y$ phonium. We specifically included species that have been moved between Arisaema, Pinellia, and Typhonium, namely A. hirsutum S. Y. Hu [Typhonium hirsutum (S. Y. Hu) J. Murata et Mayo], A. submonoicum Gagnep. [T. horsfieldii (Miq.) Steenis], and A. tripartitum Engl. [Pinellia tripartita (Blume) Schott].

Total genomic DNA was isolated from silica-dried leaves using DNeasy kits (QIAGEN, Valencia, California, USA) or the method of Doyle and Doyle (1987). DNA amplification by the polymerase chain reaction (PCR) was performed according to the protocol described in Zhang and Renner (2003). To amplify the chloroplast $\operatorname{trn} L$ intron and adjacent spacer before the $\operatorname{trn} F$ gene, we used the universal primers c, d, e, and f of Taberlet et al. (1991). The chloroplast rpl20-rps 12 intergenic spacer between the ribosomal protein genes S12 and L20 was sequenced using primers rpl20 and rps 12 of Hamilton (1999).

Amplified fragments were purified by running the entire product on a low melting-point agarose gel and then recovering the DNA with QIAquick Gel Extraction Kits (QIAGEN). Cycle sequencing of the purified PCR products used the BigDye Terminator Cycle Sequencing kit (Applied Biosystems [ABI], Norwalk, Connecticut, USA) according to the manufacturer's suggested protocol. The dye was removed by $2 \mu \mathrm{L}$ of $3 \mathrm{~mol} / \mathrm{L} \mathrm{NaOAc}(\mathrm{pH} 4.6)$ and $50 \mu \mathrm{L}$ ethanol precipitation. Samples were then run on the ABI 377 automated sequencer of the Department of Biology at the University of Missouri-St. Louis. Both strands were sequenced and used to generate consensus sequences in Sequencher (version 4.1.2, GeneCodes, Ann Arbor, Michigan, USA), which was also used for the manual sequence alignment of all sequences. Alignment was unproblematic except for a long stretch of up to 218 base pairs (bp) in the trnL intron. The aligned sequences were copied into NEXUS files for phylogenetic analysis.

Phylogenetic analyses and molecular clock dating - Tree searches were conducted with version 4.0b.10 of PAUP* (Swofford, 2002) or MrBayes version 3.0B4 (Huelsenbeck and Ronquist, 2001; Ronquist and Huelsenbeck, 2003). DNA insertions or deletions were treated as missing data and excluded from most parsimony, maximum likelihood (ML), and Bayesian analyses. Parsimony analyses used heuristic searching, 10 random sequence taxon addition replicates, with 100 trees in memory, and tree bisection-reconnection (TBR) swapping. Clade support was assessed via nonparametric bootstrapping, using PAUP. Bootstrap analyses under parsimony used 200 replicates, with closest taxon addition and one tree held in memory. The "collapse," but not the "steepest descent," option of PAUP were in effect during all searches, and character changes were interpreted under ACCTRAN optimization.

Maximum likelihood (ML) analyses were performed using the general time reversible $(\mathrm{GTR})+\mathrm{Gamma}(\mathrm{G})+\mathrm{P}_{\mathrm{inv}}$ model, which uses a discrete gamma model (in our case with four rate categories) to approximate the continuous gamma distribution (Yang, 1994; implemented in PAUP) and also assumes a proportion of invariable sites. Base frequencies were the empirically observed ones. The shape parameter of the gamma distribution can be estimated efficiently from any of numerous equally parsimonious trees because its dependence on tree topology is minor (Yang and Kumar, 1996; Sullivan et al., 1999), which greatly reduces the computational demands of maximum likelihood searches.

Bayesian analyses used the GTR $+\mathrm{G}$ model. One cold and three incre- mentally heated Markov chain Monte Carlo $(\mathrm{mcmc})$ chains were run for 100000 or 1 million cycles, with trees sampled every 10th or 100th generation, using random trees as starting points and a temperature parameter value of 0.2 (the default in MrBayes). For each data set, mcmc runs were repeated at least twice as a safeguard against spurious results. The first 5000 or 7000 trees were discarded as burn-in, and the remaining tree used to construct Bayesian consensus trees. Examination of the log-likelihoods and the observed consistency between runs suggested that these burn-in periods were sufficiently long for chains to have become stationary.

For the molecular clock dating, we first performed a likelihood ratio test (LRT; Felsenstein, 1981) that compared the likelihood scores with and without the clock assumption on one of the 26 equally parsimonious trees obtained from the combined data (excluding all gaps and rooted with just Pinellia tripartita). When the data did not reject the clock (Results), we used an ultrametric (clock-enforced) tree to obtain age estimates for the biogeographically interesting disjunctions. In addition, we used cross-validated penalized likelihood (Sanderson, 2002; implemented in the Unix shareware program r8s, http://ginger.ucdavis.edu/r8s/), because this software enables one to incorporate multiple calibration points and minimal and/or maximal ages, rather than a single, fixed calibration point, with the program then calculating the most likely ages of nodes given the remaining constraints and substitutions in the data set. Penalized likelihood is a semiparametric approach that allows different substitution rates between ancestral and descendent branches, but then reduces the resulting enormous number of more or less arbitrary alternatives by assigning a penalty that increases with the abruptness of rate change between adjacent branches. The penalty (or "smoothing" parameter) is calculated by sequentially removing part of the data (one branch at a time), reestimating the remaining model parameters, and using the fitted model parameters to predict the data that were removed (i.e., the expected number of substitutions on the pruned branch). In the case of clocklike or almost clocklike data, the smoothing value is high, resulting in invariable rates between ancestors and descendants (Sanderson, 2002). Trees were studied in TreeEdit (http://evolve.zoo.ox.ac.uk/software/TreeEdit/; Rambaut and Charleston, 2000).

A calibration for genetic distances in Arisaema comes from infructescences from the mid-Miocene Latah Formation near Spokane (18-16 [million years] ago; Knowlton, 1926) that closely match infructescences of living North American A. triphyllum.

\section{RESULTS}

Sequence data-The aligned trnL-F sequences comprised 459 nucleotide positions, of which we excluded one poly-T run. The alignment of the rpl20-rps 12 sequences, with gaps, comprised 861 nucleotides. We excluded three short regions of poly-A or poly-T runs. The aligned $t r n L$ intron sequences comprised 726 nucleotide positions, of which we excluded a TA tandem array region of $184 \mathrm{bp}$. The concatenated sequences from the three loci comprised 2048 nucleotides, of which 274 were eliminated. This matrix contained 98 (5.5\%) autapomorphic variable sites and $63(3.6 \%)$ potentially parsimonyinformative sites for 31 taxa. Under the GTR $+\mathrm{G}+\mathrm{P}_{\text {inv }}$ model, the estimated value of the gamma shape parameter was 0.877 , indicating an almost random distribution of the rates at which sites are changing.

Phylogenetic analyses-The trnL-F spacer sequences contain too little signal to yield much resolution for the 81 ingroup accessions (Fig. 2). It is apparent, however, that many species group according to section, albeit usually without statistical support. (The 11 sections recognized by Murata [1984, 1990b] are Arisaema, Clavata, Decipientia, Dochafa, Fimbriata, Franchetiana, Nepenthoidea, Pedatisecta, Sinarisaema, Tenuipistillata, and Tortuosa. All are here represented by their type species, except section Decipientia of which we could 


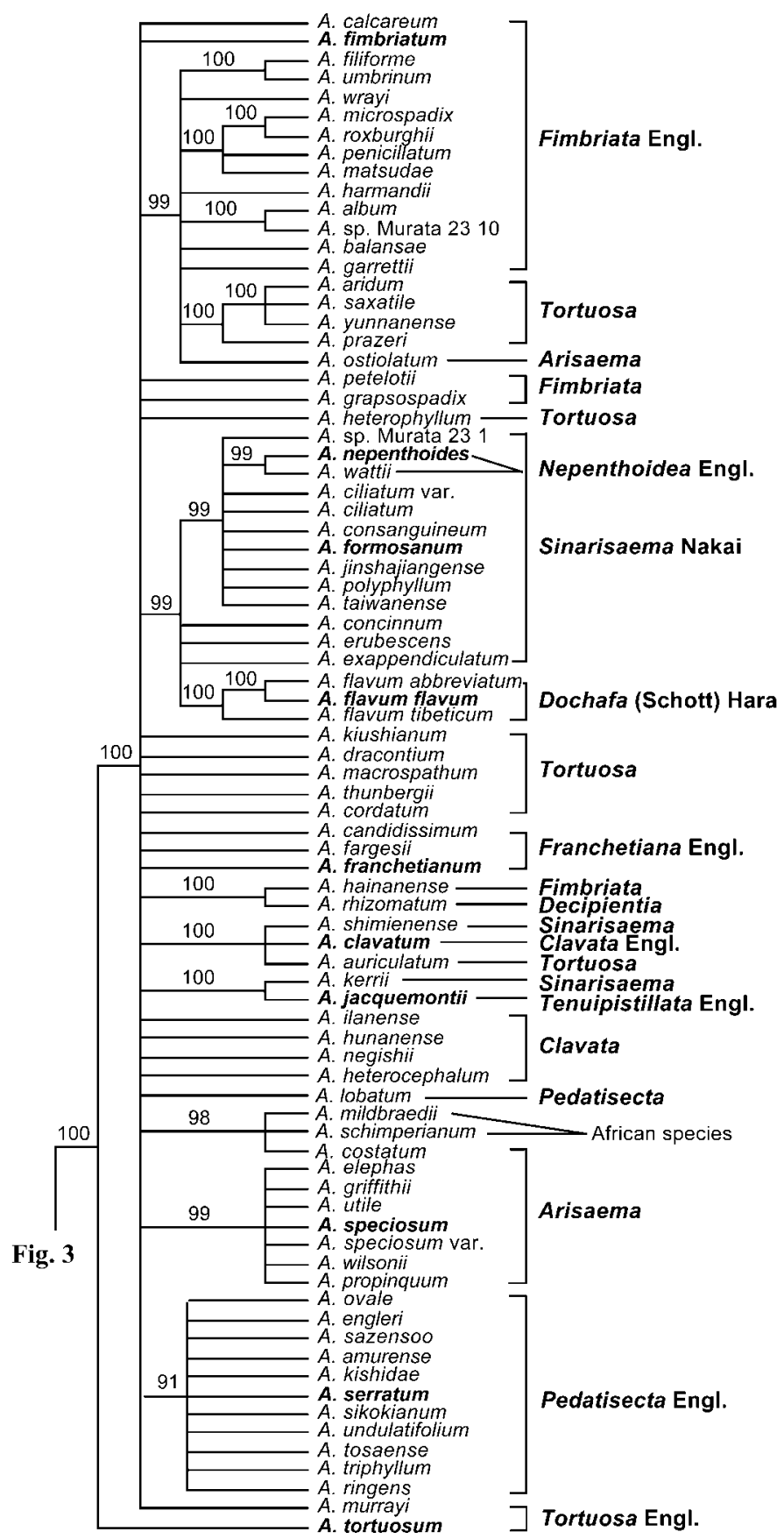

Fig. 2. Phylogeny of Arisaema obtained from trnL-F spacer sequences (459 bp) by Bayesian inference under the GTR $+\mathrm{G}$ model. Outgroups used were the same as in Fig. 3, but are here removed to save space. Posterior probabilities on branches are based on a $90 \%$ majority-rule consensus of the last 3000 of 10000 trees resulting from 100000 cycle Markov chain Monte Carlo run sampled every 10th generation. Sectional assignments of species follow Murata (1984, 1990) except for the two African species not placed with confidence; Murata's usage of section names Trisecta (= Arisaema) and Arisaema ( $=$ Nepenthoidea) has been changed to conform to an overlooked generic lectotypification (see text). Names of section types appear in boldface type. The full names of the sequenced varieties of A. ciliatum and A. speciosum are given in the Appendix (see Supplementary Data accompanying the online version of this article). not obtain type species material and instead included material of $A$. rhizomatum, the only other species placed in this section.) When we sequenced more than one accession per species (A. ciliatum, A. flavum, and A. speciosum), conspecific accessions differed by one or two substitutions in the trnL intron, the $\operatorname{trnL}-F$ spacer, or the rpl20-rps 12 spacer, with the three regions not differing consistently in information content at this level.

Combined $t r n L-F$ and $r p l 20$-rps 12 spacer sequences for species selected to represent the morphological diversity of the genus (Fig. 3) provide strong support for its monophyly and limited support for intrageneric relationships. This data set also indicates that section Tortuosa is polyphyletic (as already hinted at by the $\operatorname{trn} L-F$ spacer data; Fig. 2), with two of its species, $A$. aridum and $A$. saxatile, embedded in section Fimbriata, while the remainder of section Tortuosa forms part of a large polytomy.

The addition of trnL intron sequences (Fig. 4) to the data greatly improves the statistical support for the species groups and also shows that the three New World species of Arisaema do not form a clade.

An LRT that compared likelihood scores under the GTR + $\mathrm{G}+\mathrm{P}_{\text {inv }}$ model, with and without the clock assumption on one of the 26 equally parsimonious trees obtained from the combined data (excluding all gaps and rooting with Pinellia tripartita), did not reject the clock model $\left(\chi^{2}=\right.$ $2(3437.4240-3421.1720)=35.50, P<0.1 ; 26 \mathrm{df})$. The data could thus be modeled as clocklike. Setting the split between A. triphyllum and its Asian sister species A. amurense to 18 my old yields a substitution rate of 0.000081 substitutions per site per my (0.00146 divided by 18$)$. This gives estimates of $31 \pm 10$ my for the divergence between Nepalese-Tibetan $A$. costatum and East African A. schimperianum and of $49 \pm 12$ my for the split between Asian A. heterophyllum and the North American A. dracontium/A. macrophyllum clade. Cross-validated penalized likelihood, for which we constrained the split between $A$. triphyllum and A. amurense to minimally 18 or 20 my old (in different runs) and the root of the genus to maximally 60 my old, yielded younger ages, namely 19 my for the split between $A$. costatum and A. schimperianum and 32 my for the split between A. heterophyllum and A. dracontium/A. macrophyllum. The divergence between the diploid A. flavum subsp. flavum and its tetraploid relatives ssp. abbreviatum and tibeticum was dated to 13 my by the ultrametric clock and to 8 my by penalized likelihood.

\section{DISCUSSION}

Monophyly and relationships of Arisaema-Molecular data support Arisaema as monophyletic, suggesting that the single known synapomorphy of the genus, the sex change (Murata, 1990a, b), evolved once in the common ancestor of extant Arisaema. The only taxa that lack the sex change strategy are subspecies abbreviatum and flavum of A. flavum and both are tetraploid (Murata, 1990c). The third subspecies of A. flavum, subsp. tibeticum, is diploid and changes sex from year to year just like the remaining species in the genus. Subspecies tibeticum occurs from Bhutan to Sichuan, ssp. abbreviatum occurs from Nepal to Oman, and the typical form of ssp. flavum is thought to range from Oman, Yemen, and Saudi Arabia to Ethiopia (our material, verified by P. Boyce, however is from Pakistan). It is plausible that the two tetraploid subspecies, concomitant with their spread into drier habitats, re- 


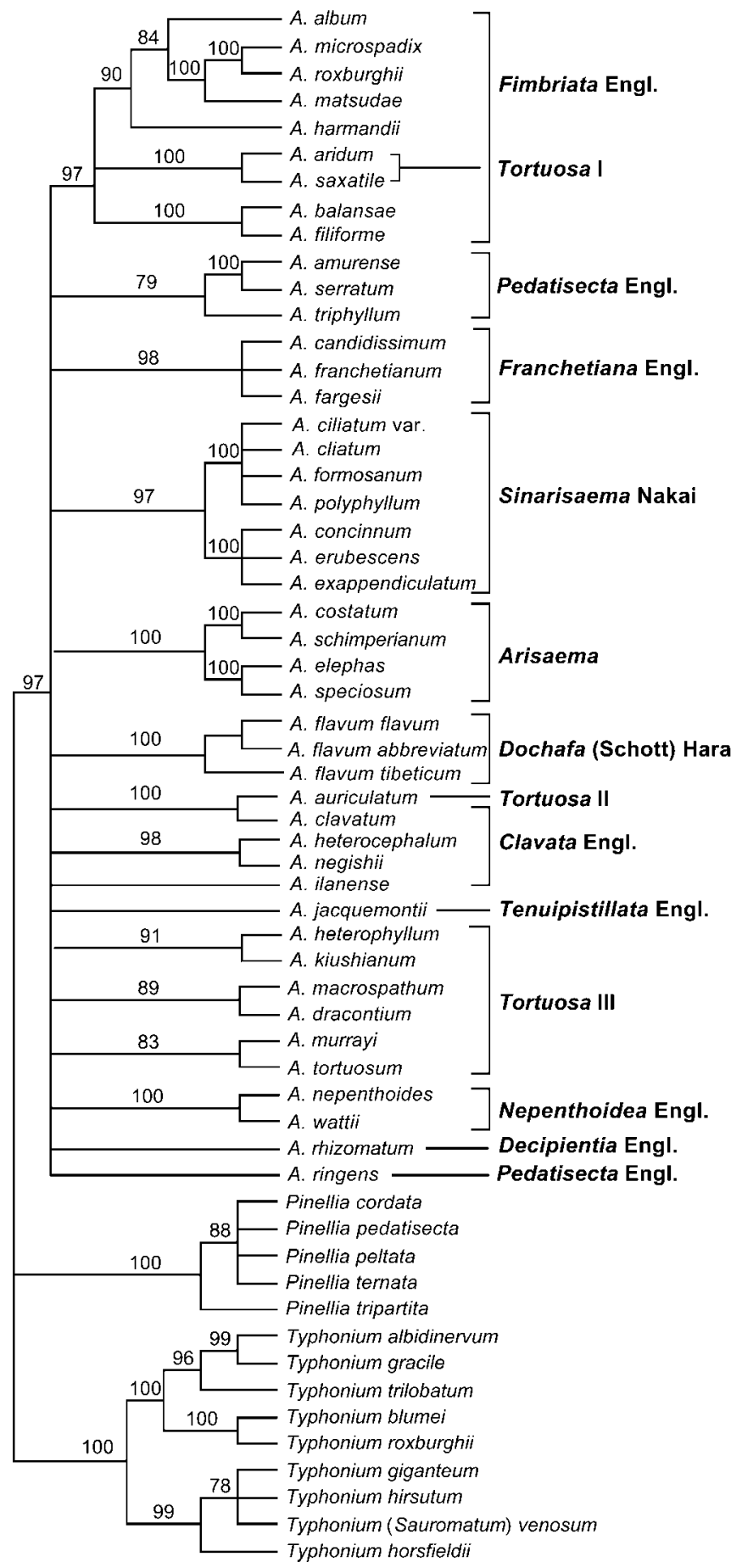

Fig. 3. Phylogeny of Arisaema and outgroups obtained from combined trnL-F and rpl20-rps 12 spacer sequences (1128 bp after removal of all gaps) by Bayesian inference under the GTR + G model. Posterior probabilites on branches are based on a $70 \%$ majority rule consensus of the last 5000 of 10000 trees resulting from a one million cycle Markov chain Monte Carlo run sampled every 100th generation.

verted from sex change and the correlated dependence on cross-pollinating insects to simultaneously bisexual spathes, which provide independence from pollinating insects because they permit automatic selfing (with self-pollen falling on receptive stigmas; Vogel and Martens, 2000). Most species of
Arisaema are pollinated by humidity-loving fungus gnats and sciarid gnats (Vogel and Martens, 2000), but the subspecies of A. flavum are unusual in offering a sugary secretion in its inflorescences (Murata et al., 1993; J. Murata, personal observations). The exact roles of insect pollination vs. automatic selfing in natural populations are unknown.

While the monophyly of Arisaema appears clear, the group's relationships to its closest relatives, Pinellia and Typhonium, remain unresolved. A study based on mitochondrial and chloroplast loci for the clade of 16 genera to which Arisaema belongs also was unable to resolve the trichotomy of Arisaema, Pinellia, Typhonium (Renner and Zhang, in press), but revealed that all genera of Areae (Arum, Biarum, Dracunculus, Eminium, Helicodiceros, and Theriophonum) are embedded in Typhonium. Based on overall phenetic resemblance, Grayum (1990, p. 682) suggested that Arisaema was sister to Areae. By contrast, Murata (1990a) saw Arisaema as sister to Pinellia, based on a uniquely shared leaf-folding pattern in which all of the lateral leaflets are folded downwards in bud. However, the downward folding is lost in three of the 11 sections of Arisaema and is found in at least one species of Typhonium transferred there from Arisaema by Murata. Additional sequencing is needed to resolve the Arisaema-Pinellia-Areae $(\sim$ Typhonium) trichotomy.

Major groups within Arisaema-Current understanding of morphological evolution in Arisaema, and most of the sectional classification, go back to Engler (e.g., 1879; our Fig. 2), who gave much weight to leaf shape (diagrams of most species' leaf shape: Gusman and Gusman, 2002). Based on new anatomical and morphological data from living material, Hara (1971), Murata (1984, 1990b), and Gusman and Gusman (2002) made major modifications to Engler's system, and the molecular data to some extent support their newly circumscribed sections, albeit without statistical support and generally with one or two species falling outside the species cluster around the type species. This is the case for sections Arisaema, Clavata, Dochafa, Fimbriata (which includes parts of Tortuosa), Franchetiana, Pedatisecta, and Sinarisaema. Section $\mathrm{Ne}$ penthoidea is supported in the combined spacer data (Fig. 3), but is not represented in the spacer + intron data (Fig. 4). Sections Decipientia and Tenuipistillata are represented by only one species (Fig. 3).

The only section that is clearly polyphyletic is Tortuosa. Tortuosa was based on pedate or rarely simple, trifoliolate, or radially 5-7-foliolate leaves and a sessile spadix appendage that is long exerted from the spathe (see keys in Murata, 1984; Gusman and Gusman, 2002). The section is especially variable in chromosome number (Watanabe et al., 1998), and all its characters also appear elsewhere in the genus. Based on our results, its traits will need to be reanalyzed or weighted differently to define a monophyletic subgroup around the type species of sect. Tortuosa.

A surprising finding is the placement of A. jinshajiangense in a clade with members of sect. Sinarisaema (Fig. 2). Arisaema jinshajiangense has leaves that originate in a quincuncial spiral and are trifoliolate (have just three leaflets), while most other species in Sinarisaema have leaves arranged in a spirodistichous spiral and with numerous radiate leaflets (all leaflets emerge from the same central point; see drawings in Gusman and Gusman, 2002, p. 298-299). Gusman and Gusman (2002, p. 144) include A. jinshajiangense in sect. Clavata but its trifoliolate leaves are also exceptional in that section, 


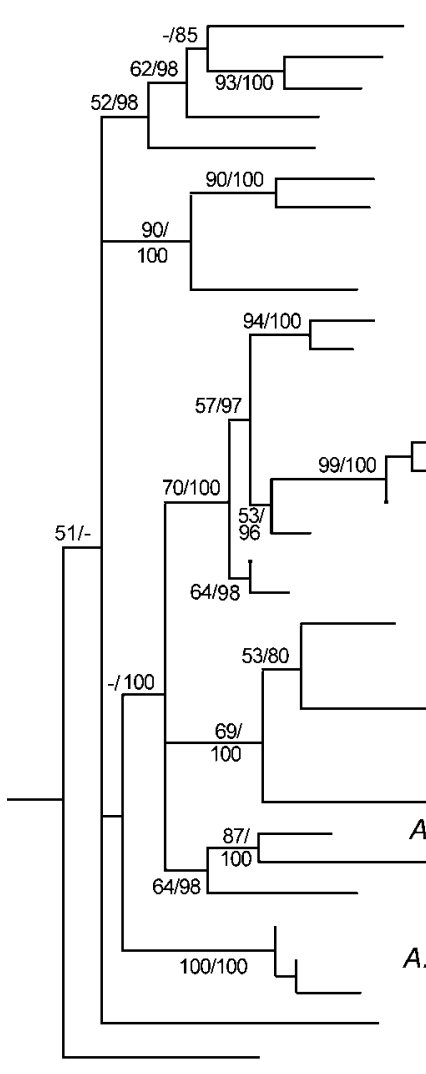

— 0.001 substitutions/site
A. album A. microspadix A. matsudae A. harmandii

A. aridum A. costatum A. schimperianum

A. speciosum A. amurense A. triphyllum $>$ NE US

A. ciliatum A. polyphyllum A. formosanum A. rhizomatum A. fargesii A. franchetianum A. dracontium
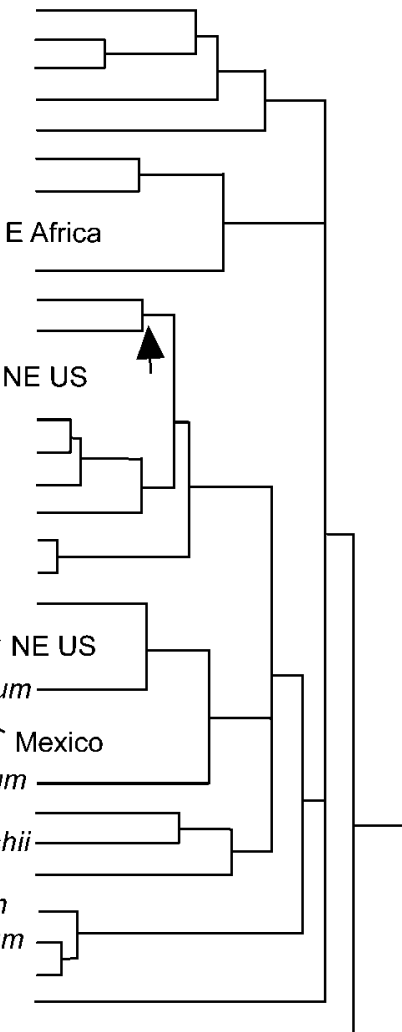

A. jacquemontii

A. tortuosum

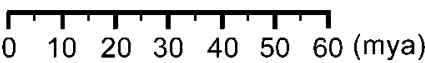

Fig. 4. One of 26 equally parsimonious trees obtained for Arisaema from combined sequences of the trnL intron, $\operatorname{trnL}-F$ spacer, and $r p l 20-r p s 12$ spacer (1577 bp excluding all gaps; compare Figs. 2 and 3 for denser ingroup and outgroup sampling). Values at branches of the left tree indicate bootstrap support from 100 replicates, followed by Bayesian posterior probabilities. Branch lengths in the left tree reflect substitutions inferred under maximum likelihood using the GTR $+\mathrm{G}+\mathrm{P}_{\text {inv }}$ model, while branch lengths in the right tree reflect modeling of substitution rates under penalized likelihood. The arrow indicates a node constrained to be 18 my old based on a fossil infructescence from Spokane, Washington, USA, that closely resembles A. triphyllum.

which usually has pedate leaves (p. 136-137). The unexpected placement of $A$. jinshajiangense with species that have quite different leaves may point to a greater plasticity in phyllotaxis than heretofore assumed for the genus.

Morphologically distinct sections that are supported by our data, such as section Arisaema, with strongly fused anther cells, a long spadix appendage that is exserted from the spathe, and erect fruiting peduncles, are usually also geographically coherent (sect. Arisaema includes 17 species that are restricted to the Sino-Himalayan region). The finding that the African species $A$. mildbraedii and A. schimperianum apparently belong in sect. Arisaema, where they are related to Nepalese/ Tibetian A. costatum, is all the more surprising. The relevant sequences all have unique motifs that differ from the remaining 74 species sequenced, which excludes contamination. The material of A. mildbraedii was obtained from Kenya, while the material of $A$. schimperianum (originally from Ethiopia) was contributed by J. Murata. The grouping of these two species with $A$. costatum is mainly due to a uniquely shared mutation from $\mathrm{A}$ to $\mathrm{C}$ at $\operatorname{trn} L-F$ nucleotide position 109 . As pointed out by Mayo and Gilbert (1986), morphological similarities exist between African/Arabian species of Arisaema and certain Nepalese species, and these authors specifically mentioned the species pair A. mildbraedii and A. consanguineum (Himalaya, northern Thailand). Our data (Fig. 2) instead show A. mildbraedii grouping with $A$. costatum and A. schimperianum, while $A$. consanguineum groups with members of Sinarisaema in agreement with its morphology (Murata, 1984). More generally, however, the data support Mayo and Gilbert's assessment of close ties between some African and Asian species (next section), and unpublished observations by Murata on cultivated A. schimperianum have revealed that its leaf arrangement is quincuncial, which would fit with the quincuncial leaf arrangement typical of section Arisaema and would provide some morphological support for the placement of this East African species in section Arisaema. Additional sampling of Indian and African species is needed for a fuller exploration of the connections between East Africa, India, and Asia.

Temporal and spatial range expansion in Arisaema-From the molecular clock estimates, the divergence between African A. schimperianum and Nepalese/Tibetan A. costatum occurred between 31 to 19 my ago, with the semiparametric approach (penalized likelihood) favoring the younger date and the ultrametric approach the older date. That penalized likelihood yields younger ages is mainly due to the constraint of the root 
node to maximally 60 my old. The justification for this constraint lies in the highly derived position of Arisaema, Pinellia, and their relatives, the Areae, in the family tree of Araceae (Cabrera et al., in press). Alternatively, one can rely on the estimates from the ultrametric approach. Either estimate fits with the considerably moister settings that existed in the southeastern parts of the Arabia peninsula during the Oligocene and Miocene (Whybrow and Hill, 1999, and references therein). Mammalian faunas of Afro-Arabia underwent a marked transition near the Oligocene/Miocene boundary at approximately 24 my, when many endemic taxa were replaced by migrants from the Northern Hemisphere (Kappelman et al., 2003). Clearly, these age estimates should be taken with a grain of salt because they are influenced by our taxon sampling density, which as discussed is lowest for Indian and African species.

The problem of taxon sampling is less acute in our placement of North American Jack-in-the-pulpit, A. triphyllum, and green dragon, A. dracontium and its Mexican relative, A. macrospathum. Arisaema triphyllum belongs to section Pedatisecta, with some 32 species endemic in China, Korea, and Japan, and is most similar to the Asian A. amurense from which it can be distinguished only by details of the inflorescence (Murata, 1984). Arisaema dracontium and A. macrospathum belong in section Tortuosa, which in addition has one species in India and 11 in China, Korea, and Japan (three extending into Taiwan, Thailand, and Burma). American A. dracontium and Mexican A. macrospathum are especially similar to Asian A. heterophyllum (Engler, 1879, p. 546-548 and more recent authors). The placement of the three American species in different Asian clades by the molecular data agrees with their traditional morphological placement in different sections and is unlikely to change with additional sampling. The 18-my-old fossil Arisaema infructescence discovered near Spokane (Knowlton, 1926) further supports that Arisaema ranged across Beringia, possibly into the Early Miocene, although the fossil of course does not indicate when connections between Asian and American populations were severed. A new insight from the molecular clock estimates is that the origin of today's A. dracontium/A. macrophyllum clade may date back to the Oligocene (whether estimated under semiparametric or ultrametric clocks) and may be quite a bit older than the A. triphyllum line. Arisaema is not the only genus inferred to have crossed Beringia repeatedly; additional Asiancentered genera with multiple links to North America include Magnolia (Qiu et al., 1995), Nyssa (Wen and Stuessy, 1993), Panax (Wen, 2001), Trillium (Kato et al., 1995), and Aralia (Wen, 1999, for a review).

Future work in the genus will depend on the addition of a suitably fast-evolving, perhaps nuclear, gene region. In our experience and that of others, working with the internal transcribed spacer region (ITS) in Arisaema is problematic (Buzgo et al., 2002), with multiple paralogous copies commonly found as a result of slow or ineffective concerted evolution.

\section{LITERATURE CITED}

BIERZYCHUdeK, P. 1984. Determinants of gender in Jack-in-the-pulpit: the influence of plant size and reproductive history. Oecologia 65: 14-18.

BuZgo, M., Y.-L. QIU, AND M. W. Chase. 2002. ITS in Arisaema (Araceae). AIBS abstract, published at http://www.botany2002.org/viewer.shtml.

Cabrera, L. I., G. A. Salazar, M. W. Chase, and S. J. Mayo. In press. Phylogenetics of Araceae and Lemnaceae: evidence from multiple plastid DNA data sets. Proceedings of the Monocots III meeting, Rancho Santa Ana Botanical Garden, Claremont, California, USA.
Charnov, E. L., AND J. Bull. 1977. When is sex environmentally determined? Nature 266: 828-830.

DoYLE, J., AND J. DOYLE. 1987. A rapid DNA isolation procedure for small quantities of fresh leaf tissue. Phytochemical Bulletin 19: 11-15.

Engler, A. 1879. Araceae. In A. and C. de Candolle. [eds.], Monographieae Phanerogamarum, vol. 2, 1-681. Wolf and Sons, Munich, Germany.

FelsensteIn, J. 1981. Evolutionary trees from DNA sequences: a maximum likelihood approach. Journal of Molecular Evolution 17: 368-376.

Freeman, D. C., K. T. Harper, AND E. L. Charnov. 1980. Sex change in plants: old and new observations and new hypotheses. Oecologia 47: 222-232.

Grayum, M. H. 1990. Evolution and phylogeny of the Araceae. Annals of the Missouri Botanical Garden 77: 628-697.

Gusman, G. 2000. Observations on Arisaema macrospathum Benth. (Araceae). Aroideana 23: 41-47.

Gusman, G., And L. Gusman. 2002. The genus Arisaema. A monograph for botanists and nature lovers. Ganter Verlag, Ruggell, Liechtenstein.

Hamilton, M. B. 1999. Four primer pairs for the amplification of chloroplast intergenic regions with intraspecific variation. Molecular Ecology 8: $521-523$.

HARA, H. 1971. A revision of the eastern Himalayan species of the genus Arisaema (Araceae). In H. Hara [ed.], Flora of eastern Himalaya, 2nd Report, 321-354. University of Tokyo Press, Tokyo, Japan.

HetTerscheid, W. L. A., AND P. C. Boyce. 2000. A reclassification of Sauromatum Schott and new species of Typhonium Schott (Araceae). Aroideana 23: 48-55.

Hetterscheid, W. L. A., D. Sookchaloem, and J. Murata. 2001. Typhonium (Araceae) of Thailand: new species and a revised key. Aroideana 24: 30-55.

HuelsenbeCK, J. P., AND F. R. Ronquist. 2001. MrBayes: Bayesian inference of phylogeny. Bioinformatics 17: 754-755.

KAPPELMAN, J., ET AL. 2003. Oligocene mammals from Ethiopia and faunal exchange between Afro-Arabia and Eurasia. Nature 426: 549-552.

Kato, H., S. Kawano, R. Terauchi, M. Ohara, and F. H. Utech. 1995. Evolutionary biology of Trillium and related genera (Trilliaceae). I. Restriction site mapping and variation of chloroplast DNA and its systematic implications. Plant Species Biology 10: 17-29.

Knowlton, F. H. 1926. Flora of the Latah formation of Spokane, Washington, and Coeur d'Alene, Idaho. U.S. Geological Survey, Reston, Virginia. Professional Paper 140A: 1-81.

LI, H. 1981. Himalayas-Hengduan Mountains-the center of distribution and differentiation of the genus Arisaema. In Li Dongsheng [ed.], Geological and ecological studies of Qinghai-Xizang Plateau, vol. 2, 1321-1327. Science Press, Beijing, China.

Li, H., G. Zhu, and J. Murata. In press. Arisaema. In C. Y. Wu and P. Raven [eds.], Flora of China, vol. 00, 00-00. Science Press, Beijing, China and Missouri Botanical Garden, St. Louis, Missouri, USA.

MAYO, S. J. 1993. Aspects of aroid geography. In W. George and R. Lavocat [eds.], The Africa-South America connection, 44-58. Clarendon Press, Oxford, UK.

Mayo, S. J., AND N. G. Gilbert. 1986. A preliminary revision of Arisaema (Araceae) in tropical Africa and Arabia. Kew Bulletin 41: 261-278.

MurAtA, J. 1984. An attempt at an infrageneric classification of the genus Arisaema (Araceae). Journal of the Faculty of Science, University of Tokyo, section 3, Botany 13: 431-482.

Murata, J. 1990a. Developmental patterns of pedate leaves in tribe Areae (Araceae-Aroideae) and their systematic implication. Botanical Magazine (Tokyo) 103: 339-343.

Murata, J. 1990b. Present status of Arisaema systematics. Botanical Magazine (Tokyo) 103: 371-382.

Murata, J. 1990c. Three subspecies of Arisaema flavum (Forssk.) Schott (Araceae). Japanese Journal of Botany 65: 65-73.

Murata, J., C. Ren, H. Murata, and S. Ogawa. 1993. Arisaema flavum, a honey-producing Araceae. Abstract 1203, 15th International Botanical Congress, Yokohama, Japan.

Qiu, Y.-L., M. W. Chase, AND C. R. Parks. 1995. A chloroplast DNA phylogenetic study of the eastern Asia-eastern North America disjunct section Rytidospermum of Magnolia (Magnoliaceae). American Journal of Botany 82: 1582-1588.

Rambaut, A. M., and M. Charleston. 2000. TreeEdit version 1.0 alpha 4-61. Computer program available at http://evolve.zoo.ox.ac.uk/ software.html?id=TreeEdit University of Oxford, Oxford, UK.

Renner, S. S., AND L.-B. Zhang. In press. Biogeography of the Pistia clade 
(Araceae) based on cp and mt DNA sequences and Bayesian divergence time inference. Systematic Biology.

Ronquist, F., AND J. P. HuelsenbeCK. 2003. MrBayes 3: Bayesian phylogenetic inference under mixed models. Bioinformatics 19: 1572-1574.

SANDERS, L. L., AND C. J. BURK. 1992. A naturally-occurring population of putative Arisaema triphyllum subsp. stewardsonii $\times$ A. dracontium hybrids in Massachusetts. Rhodora 94: 340-347.

SANDERSON, M. J. 2002. Estimating absolute rates of molecular evolution and divergence times: a penalized likelihood approach. Molecular Biology and Evolution 19: 101-109.

Schlessman, M. A. 1988. Gender diphasy ("sex choice"). In J. Lovett Doust and L. Lovett Doust [eds.], Plant reproductive ecology: patterns and strategies, 139-153. Oxford University Press, New York, New York, USA.

Sriboonma, D., J. Murata, AND K. Iwatsuki. 1994. A revision of $T y$ phonium (Araceae). Journal of the Faculty of Science, University of Tokyo, section 3, Botany 15: 255-313.

Sullivan, J., D. L. Swofford, AND G. J. P. NAYlor. 1999. The effect of taxon sampling on estimating rate heterogeneity parameters of maximum-likelihood models. Molecular Biology and Evolution 16: 13471356.

SwOFForD, D. L. 2002. PAUP*. Phylogenetic analysis using parsimony (*and other methods), version 4. Sinauer Associates, Sunderland, Massachusetts, USA.

Taberlet, P., L. Gielly, G. Pautou, And J. Bouvet. 1991. Universal primers for amplification of three non-coding regions of chloroplast DNA. Plant Molecular Biology 17: 1105-1109.
Vogel, S., AND J. Martens. 2000. A survey of the function of the lethal kettle traps of Arisaema (Araceae), with records of pollinating fungus gnats from Nepal. Botanical Journal of the Linnean Society 133: 61100.

Watanabe, K., T. Kobayashi, and J. Murata. 1998. Cytology and systematics in Japanese Arisaema (Araceae). Journal of Plant Research 111: 509-521.

WEN, J. 1999. Evolution of eastern Asian and eastern North American disjunct distributions in flowering plants. Annual Review of Ecology and Systematics 30: 421-455.

WEN, J. 2001. Evolution of the Aralia-Panax complex (Araliaceae) as inferred from nuclear ribosomal ITS sequences. Edinburgh Journal of Botany 58: 183-200.

Wen, J., AND T. F. StUESSY. 1993. The phylogeny and biogeography of Nyssa (Cornaceae). Systematic Botany 18: 68-79.

Whybrow, P. J., AND A. Hill [EDs.]. 1999. Fossil vertebrates of Arabia. Yale University Press, New Haven, Connecticut, USA.

YANG, Z. 1994. Maximum likelihood phylogenetic estimation from DNA sequences with variable rates over sites: approximate methods. Journal of Molecular Evolution 39: 306-314.

YANG, Z., AND S. KumAR. 1996. Approximate methods for estimating the pattern of nucleotide substitution and the variation of substitution rates among sites. Molecular Biology and Evolution 13: 650-659.

ZhANG, L.-B., AND S. S. RENNER. 2003. The deepest splits in Chloranthaceae as resolved by chloroplast sequences. International Journal of Plant Sciences 164(5 Suppl.): S383-S392. 